\title{
TUMORES MALIGNOS DO INTESTINO DELGADO
}

\author{
MALIGNANT TUMORS OF THE SMALL INTESTINE
}

\author{
Marcelo Fernandes Rangel, TCBC-PB ${ }^{1}$ \\ Marcos Valério Maia da Silva, ACBC-PB ${ }^{2}$ \\ Manoel Jeovah Colaço Fernandes, TCBC-PB ${ }^{3}$ \\ Mário Augusto Souto Ferreira ${ }^{4}$ \\ Leonardo Pires de Sá Nóbrega ${ }^{5}$ \\ Marcelo Gonçalves Souza ${ }^{6}$
}

\begin{abstract}
RESUMO: Os tumores do intestino delgado são raros e a maioria das lesões neoplásicas sintomáticas é maligna. Os neoplasmas benignos são um pouco mais freqüentes e ambos estão relacionados a um diagnóstico difícil, pois determinam queixas abdominais inespecíficas, comuns a uma grande variedade de afecções digestivas. Exames de imagem e endoscópicos podem ser úteis para o diagnóstico, mas freqüentemente não são conclusivos. Para os blastomas primários, a ressecção cirúrgica é a opção de escolha, porém, para os metastáticos, a terapêutica operatória deve ser reservada para os casos complicados por obstrução, hemorragia ou perfuração. O presente estudo tem por finalidade analisar retrospectivamente 13 casos de lesões malignas do intestino delgado, num período de 28 anos. Verificou-se maior incidência de tumores primários $(69,2 \%)$ e de linfomas (30,7\%). Entre os secundários, as mestástases por adenocarcinoma foram as mais freqüentes $(15,4 \%)$. Enterectomia segmentar foi o procedimento cirúrgico mais realizado $(84,6 \%)$ e a mortalidade hospitalar foi de $15,4 \%$. A sobrevida de cinco anos foi nula para os pacientes portadores de metástases, enquanto que para os primários foi de $44,4 \%$, sugerindo um melhor prognóstico para as neoplasias primitivas, independentemente do tipo histológico da neoplasia.
\end{abstract}

Descritores: Intestino delgado; Neoplasias malignas; Tratamento cirúrgico; Sobrevida.

\section{INTRODUÇÃO}

Em todas as faixas etárias, os tumores do intestino delgado são raros, correspondendo a cerca de $1 \%$ a $6 \%$ de todas as neoplasias do trato gastrointestinal ${ }^{1}$.

Os neoplasmas benignos são um pouco mais comuns do que os malignos, e ambos estão relacionados a um diagnóstico difícil, pois determinam queixas vagas e inespecíficas. Por isso, sintomas e sinais como dor abdominal, episódios de oclusão intestinal, sangramento digestivo, perda de peso e massa palpável são manifestações comuns a uma grande variedade de afecções digestivas e induzem a um retardo no esclarecimento dessas lesões ${ }^{2}$.

Exames de laboratório, de imagem e endoscópicos podem ser úteis na propedêutica complementar, mas freqüentemente não são definitivos para confirmação da hipótese diagnóstica.

O princípio geral do tratamento dos blastomas malignos primários do intestino delgado é a ressecção com margens cirúrgicas livres, às vezes associada à linfadenectomia locorregional, na dependência do tipo histológico e do estadiamento cirúrgico da neoplasia ${ }^{3}$.

1. Professor Adjunto - Doutor do Departamento de Cirurgia do C.C.S. da UFPB. Titular do Colégio Brasileiro de Cirurgia Digestiva. Chefe do Serviço de Cirurgia Abdominal do Hospital de Câncer Napoleão Laureano.

2. Cirurgião do Serviço de Cirurgia Abdominal do Hospital de Câncer Napoleão Laureano e do Hospital Universitário da UFPB.

3. Professor Adjunto - Mestre do Departamento de Cirurgia do C.C.S. da UFPB. Cirurgião do Hospital de Câncer Napoleão Laureano.

4. Interno do Curso de Medicina do CCS da UFPB.

5. Aluno de Graduação do Curso de Medicina do CCS da UFPB. Bolsista do PIBIC/CNPq/UFPB.

6. Aluno de Graduação do Curso de Medicina do CCS da UFPB. Monitor da Disciplina de Bases da Técnica Cirúrgica.

Recebido em 24/4/2000

Aceito para publicação em 5/9/2000

Trabalho realizado no Serviço de Cirurgia Abdominal do Hospital de Câncer Napoleão Laureano - João Pessoa/PB. 
Nos casos de tumores metastáticos, a conduta é preferencialmente conservadora, reservando-se a terapêutica cirúrgica principalmente para as complicações obstrutivas e hemorrágicas ${ }^{4}$.

A raridade dessas neoplasias e a experiência com 13 casos tratados num período de 28 anos motivam a presente publicação.

\section{MÉTODOS}

Foram analisados retrospectivamente os prontuários de 13 pacientes portadores de tumores malignos do intestino delgado, operados no Serviço de Cirurgia Abdominal do Hospital de Câncer Napoleão Laureano, em João Pessoa - PB, no período de 1965 a 1993 . Sete pacientes $(53,9 \%)$ eram do sexo feminino, e os extremos de idade foram de quatro e 65 anos (média de 40,3 anos). As manifestações clínicas mais comuns foram dor abdominal em nove casos $(69,2 \%)$, perda de peso em sete $(53,8 \%)$, massa palpável em quatro $(30,7 \%)$ e sangramento digestivo em dois $(15,4 \%)$. Porém, sintomas obstrutivos acometeram a totalidade dos pacientes.

Exames de imagem e endoscópicos realizados em poucos casos, não permitiram avaliações.

Todos os pacientes foram submetidos à laparotomia exploradora por incisão mediana, e a conduta operatória foi definida após exploração e estadiamento cirúrgicos.

\section{RESULTADOS}

Foram identificados nove casos de tumores primários e quatro metastáticos. Em sete pacientes $(53,9 \%)$ a lesão localizava-se no jejuno e quatro eram ileais $(30,7 \%)$. Em dois casos $(15,4 \%)$ havia carcinomatose peritoneal, não permitindo determinar a localização exata da lesão.

O diagnóstico só foi definido durante a abordagem cirúrgica e os procedimentos adotados incluíram: enterectomia segmentar em $84,6 \%$ dos casos, exploração com biópsia em um $(7,7 \%)$ e orquiectomia mais laparotomia com biópsia em outro paciente $(7,7 \%)$. As complicações pós-operatórias observadas em três pacientes distintos (23\%) apontaram para infecção da ferida operatória, broncopneumonia e diarréia. A mortalidade hospitalar atingiu a taxa de $15,4 \%$. O estudo anatomopatológico das peças ressecadas e das biópsias identificou nove tumores primários $(69,2 \%)$, sendo quatro linfomas do tipo ocidental $(30,7 \%)$, três sarcomas (um histiocítico e dois leiomiossarcomas) correspondendo a $23 \%$ dos casos, e dois adenocarcinomas $(15,4 \%)$. Quatro tumores metastáticos $(30,7 \%)$ foram reportados, correspondendo a dois adenocarcinomas $(15,4 \%)$, um carcinoma epidermóide $(7,7 \%)$ e um carcinoma anaplásico (Tabela 1). Quimioterapia adjuvante foi instituída em oito oportunidades $(61,5 \%)$, porém em esquemas distintos, inviabilizando qualquer avaliação. A sobrevida global de cinco anos foi de $30,7 \%$, sendo nula para os portadores de metástases, enquanto que para as neoplasias primárias atingiu o índice de $44,4 \%$.
Tabela 1

Tipo histológico das neoplasias em 13 casos de tumores malignos do intestino delgado.

\begin{tabular}{l|c|c}
\hline Tipo Histológico & $N^{o}$ & $\%$ \\
\hline Linfoma tipo ocidental & 4 & 30,7 \\
Leiomiossarcoma & 2 & 15,4 \\
Adenocarcinoma & 2 & 15,4 \\
Adenocarcinoma metastático & 2 & 15,4 \\
Sarcoma histiocítico & 1 & 7,7 \\
Carcinoma anaplásico metastático & 1 & 7,7 \\
Carcinoma epidermóide metastático & 1 & 7,7 \\
\hline Total & 13 & 100,0 \\
\hline
\end{tabular}

\section{DISCUSSÃO}

No intestino delgado, os tumores benignos são um pouco mais comuns do que os malignos, e os de origem epitelial figuram entre os de maior frequiência. Os pólipos adenomatosos e vilosos podem ser sésseis ou pediculados. Estes últimos estão associados a sangramento e obstrução intestinal (intussuscepção), bem como a um baixo e controvertido potencial de malignização. $\mathrm{O}$ íleo, o duodeno e o jejuno abrigam, por ordem de frequiência, a maioria dessas lesões ${ }^{4}$.

A linhagem não epitelial das neoplasias benignas do intestino delgado inclui os leiomiomas, os linfomas, os fibromas e os neurofibromas. Com exceção dos linfomas e dos neurofibromas, que são vistos com maior freqüência no íleo, os demais incidem preferencialmente no jejuno. Sintomas obstrutivos e principalmente sangramento por necrose ou ulceração da mucosa podem ser observados em todas as modalidades, e o diagnóstico diferencial com lesões malignas muitas vezes só é realizado durante a exploração cirúrgica.

As malignidades do intestino delgado aparecem com distribuição distinta em algumas séries. Nos pacientes mais jovens o adenocarcinoma desponta com 30\% a 50\% dos casos, porém, nos idosos, o tumor carcinóide tem incidência ligeiramente maior. Em outras séries, encontrou-se maior prevalência de linfomas, variável de $20 \%$ a $65 \%$.

A maioria das lesões neoplásicas sintomáticas do intestino delgado é maligna. Os adenocarcinomas são encontrados no duodeno e jejuno proximal em cerca de 50\% das oportunidades, e estão associados a algumas condições prémalignas, como a doença de Crohn, a polipose adenomatosa familiar e a síndrome de Gardner. Quando localizados no íleo, manifestam-se clinicamente com dor, náuseas e distensão abdominal. A suspeita diagnóstica geralmente é feita por meio de exame radiológico contrastado e a confirmação pela exploração cirúrgica.

O tratamento de escolha é a ressecção do tumor com margens de segurança de pelo menos $5 \mathrm{~cm}$, associada à linfadenectomia locorregional, procedimento adotado em quatro pacientes desta série (dois adenocarcinomas primá- 
rios e dois metastáticos). A polipectomia é recomendada quando a lesão maligna se assesta em um pólipo menor do que $2 \mathrm{~cm}$. A duodenopancreatectomia está indicada nos comprometimentos periampulares e a hemicolectomia direita quando localizadas no íleo terminal. O mau prognóstico dessas neoplasias está relacionado com o estádio avançado quando diagnosticadas ${ }^{6}$. Entre $50 \%$ a $80 \%$ já apresentam metástases a distância na ocasião da primeira abordagem. A quimioterapia e a radioterapia, conforme demonstraram nossos resultados, têm valor terapêutico pouco significativo.

O leiomiossarcoma é o tumor de maior incidência no intestino delgado. A manifestação clínica mais comum é o sangramento e, mais raramente, pode evoluir com oclusão intestinal. $\mathrm{O}$ tratamento cirúrgico fundamenta-se na ressecção local ou enterectomia segmentar. Quimioterapia ou radioterapia externa podem ser utilizadas como terapêutica adjuvante, porém os resultados não são conclusivos. A sobrevida de cinco anos para o leiomiossarcoma do intestino delgado mantém-se em torno de $35 \%$ a $50 \%$ para os casos submetidos à ressecção radical ${ }^{4}$.

Os tumores primários de células linfóides do intestino delgado apresentam-se, comumente, sob a forma de lesão localizada e restrita a um segmento do íleo. Esta forma de apresentação caracteriza o linfoma do tipo ocidental, enquanto que o envolvimento difuso do intestino delgado é característica da variedade mediterrânea. Clinicamente podem estar associados a surtos de oclusão intestinal (intussuscepção), má-absorção, perda ponderal e dor abdominal.

Para os linfomas do tipo ocidental, a conduta mais efetiva é a ressecção cirúrgica de toda a massa tumoral, reservando-se a quimioterapia e/ou radioterapia para as lesões irressecáveis. Nos casos de linfomas tipo mediterrâneo, a quimioterapia é o tratamento de eleição, permane- cendo a indicação de exerese cirúrgica apenas para pacientes com doença localizada.

O tumor carcinóide do intestino delgado é raro, porém apresenta o melhor prognóstico, com taxas de sobrevida global de cinco anos de aproximadamente 50\%. A localização preferencial é o íleo distal e a incidência de metástases está diretamente relacionada com o tamanho da lesão primária; sua ocorrência gira em torno de $90 \%$ dos casos nas lesões maiores do que $2 \mathrm{~cm}^{7}$. A síndrome carcinóide está presente em apenas $10 \%$ das oportunidades e a ressecção cirúrgica representa a melhor opção terapêutica.

A extensão direta de tumores gástricos, pancreáticos, colônicos, ovarianos, uterinos, renais e, por via hematogênica, os melanomas, dá origem às metástases do intestino delgado, na maioria das vezes.

O quadro clínico geralmente é característico, manifestando-se sob a forma de oclusão intestinal intermitente. O diagnóstico é confirmado por exames de imagem, incluindo urografia excretora, ultra-sonografia, tomografia computadorizada e angiografia ${ }^{8}$. O tratamento deve ser individualizado e reservado para as lesões sintomáticas, havendo indicação da abordagem cirúrgica para as situações complicadas por obstrução, hemorragia ou perfuração ${ }^{4}$.

A análise desta casuística revelou que apenas um dos 13 pacientes tinha idade superior a 60 anos, e que o linfoma teve a maior prevalência. Entre os tumores metastáticos mais freqüentes, a próstata e o estômago foram os locais primários do adenocarcinoma.

A taxa de sobrevida de cinco anos, nos portadores de metástases, foi nula, enquanto que para os neoplasmas primários atingiu o índice de $44,4 \%$. Assim, para as malignidades do intestino delgado, pode-se inferir que o melhor prognóstico está relacionado com os tumores primários, independentemente da faixa etária do paciente ou do tipo histológico da neoplasia.

\begin{abstract}
Small bowel tumors are rare, and the majority of symptomatic lesions are malign. Benign tumors are a more frequent. Both benig and maligmant mors are associated with difficult diagnosis, because of atipic abdominal complaints, common to a large variety of digestives affections. Endoscopic and imaging examination may have diagnostic, but frequently are inconclusives. For primary tumors, the surgical resection is the treatment of choice, but for metastatic, tumors surgical resection has to be reserved for the cases complicated by obstruction, bleeding or perforation. This study has aim to analyse retrospectively 13 cases of small bowel maligns tumors in a period of 28 years. A higher incidence of primary tumors $(69,2 \%)$, and linfomas $(30,7 \%)$ were observed. Among metastatic tumors, the adenocarcinoma was the most frequent (15,4\%). Segmental enterectomy was the most common surgical procedure (84,6\%), and hospital mortality was $15,4 \%$. Survival rate within five years was zero for metastatic tumors, while for primary lesions was $44,4 \%$, suggesting a better prognostis for primary lesions, independently from the histologic type of neoplasms.
\end{abstract}

Key Words: Small bowel; Malignant neoplasms; Surgical treatment; Survival.

\title{
REFERENNCIAS
}

1. Olmstedw, W. - Tumores of small intestine with or no malignant predisposition. Review of literature. Gastroint. Radiol. 1987; 12: $131-3$.
2. Dorman, J. E.; Floyd, E.; Cohn, I. Jr. - Malignant neoplasms of the small bowel. Am. J. Surg. 1967; 113: $131-4$. 
3. Rangel, M.F.; Kunzle, J. E.; Simões, J.B.R. et al: Generalidades sobre câncer do tubo digestivo. In: Petroianu, A.; Pimenta, L. G. (Eds) Clínica e Cirurgia Geriátrica. $1^{\text {a }}$ Edição, Guanabara Koogan, Rio de Janeiro, 1999, pp. 275 - 283.

4. Bell, J.L.; Edwards, M.J.; Pollock, R.E. - Tumores do intestino delgado. In: Coelho, J.C.U. (Ed) Aparelho digestivo: Clínica e Cirurgia. $2^{a}$ Edição. Medsi, Rio de Janeiro, 1996, pp. 581-89.

5. Rosenthal, R. A. - Distúrbios do intestino delgado e hérnias da parede abdominal no paciente idoso. Cli. Cir. Am. Norte 1994; 2: 293 - 325.

6. Jonhson, A.M. ; Harman, L. K. ; Hanks, J.B. - Primary small bowel malignancies. Ann. Surg. 1985; 51: 31-6.
7. Creutzfeld, W.; Stockmann, F. - Carcinoids and carcinoid syndrome. Am. J. Med. 1987; 32 - 9.

8. Dudiak, K. M. ; Johnson, C.D. ; Stephens, D.H. - Primary tumors of the small intestine. A.J.R. 1989; 152:995-97.

Endereço para Correspôndência:

Dr. Marcelo Fernandes Rangel

Rua Clodoaldo Gouveia, 66 - Centro.

58013-370 - João Pessoa - PB 